\title{
Nickel-Catalyzed Conversion of Amides to Carboxylic Acids
}

\author{
Rachel R. Knapp, Ana S. Bulger, and Neil K. Garg* \\ Department of Chemistry and Biochemistry, University of California \\ Los Angeles, California 90095
}

Supporting Information - Table of Contents

Materials and Methods

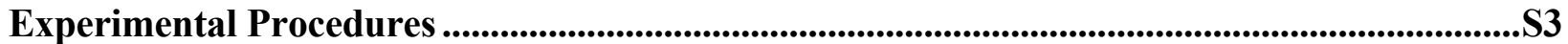

A. Initial Experiments for Amide Hydrolysis. ...................................................................................

B. Optimization and Relevant Control Experiments. ..........................................................................S3

C. Scope of Methodology .........................................................................................................................55

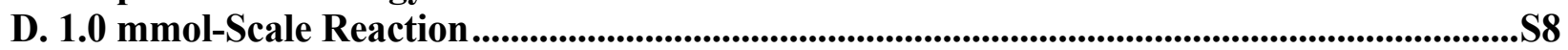

E. Competition Experiments ......................................................................................................................S9

F. Selective Cleavage of Aryl Amide in the Presence of an Ester .................................................S10

References.......................................................................................................................................................S12

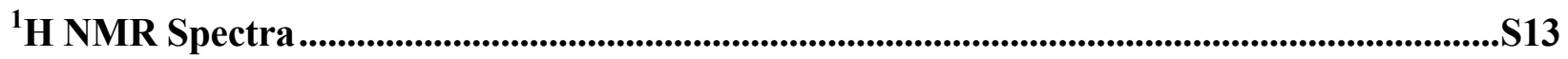

SFC Traces ...........................................................................................................................................................S21 
Materials and Methods. Unless stated otherwise, reactions were conducted in flame-dried glassware under an atmosphere of nitrogen and commercially obtained reagents were used as received. (Trimethylsilyl)ethanol (7) was obtained from Combi-Blocks and distilled and degassed by sparging with $\mathrm{N}_{2}$ for $\geq 10$ min prior to use. $\mathrm{Ni}(\operatorname{cod})_{2}$ and $\mathrm{SIPr}$ were obtained from Strem Chemicals and degassed by sparging with $\mathrm{N}_{2}$ for $\geq 10$ min prior to use. Toluene was obtained from Fisher Scientific and purified by distillation over $\mathrm{CaH}_{2}$ then taken through five freeze-pump-thaw cycles prior to use. 1,3,5-Trimethoxybenzene was obtained from Alfa Aesar and used as received. Reaction temperatures were controlled using an IKAmag temperature modulator, and unless stated otherwise, reactions were performed at room temperature (approximately $23{ }^{\circ} \mathrm{C}$ ). Thin-layer chromatography (TLC) was conducted with EMD gel 60 F254 pre-coated plates $(0.25 \mathrm{~mm}$ for analytical chromatography and $0.50 \mathrm{~mm}$ for preparative chromatography) and visualized using a combination of UV, anisaldehyde, iodine, and potassium permanganate staining techniques. Silicycle Siliaflash P60 (particle size 0.040-0.063 mm) was used for flash column chromatography. ${ }^{1} \mathrm{H}$ NMR spectra were recorded on Bruker spectrometers $(400,500$, and $600 \mathrm{MHz})$ and are reported relative to residual solvent signals. Data for ${ }^{1} \mathrm{H}$ NMR spectra are reported as follows: chemical shift $(\delta \mathrm{ppm})$, multiplicity, coupling constant $(\mathrm{Hz})$, integration. Determination of enantiopurity was carried out on a Mettler Toledo SFC (supercritical fluid chromatography) using a Daicel ChiralPak IA-3 column. Optical rotations were measured with a Rudolph Autopol III Automatic Polarimeter.

Note: Supporting information for the syntheses of amides $\mathbf{1}, \mathbf{4}, \mathbf{1 4 a}-\mathbf{d}, \mathbf{1 8}$, and $\mathbf{2 1 - 2 4 ^ { 1 }}$ have been published and spectral data match those previously reported. 


\section{Experimental Procedures}

\section{A. Initial Experiments for Amide Hydrolysis.}

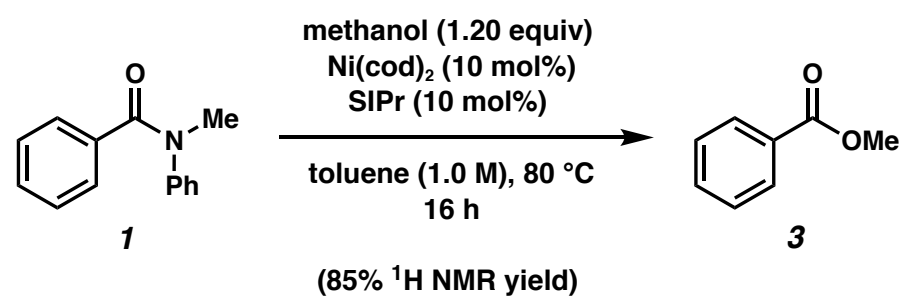

Representative Procedure for Figure 2. A 2-dram vial containing amide 1 (40.9 mg, 0.194 mmol, 1.00 equiv) and a magnetic stir bar was charged with $\mathrm{Ni}(\mathrm{cod})_{2}(5.33 \mathrm{mg}, 0.019 \mathrm{mmol}$, $10.0 \mathrm{~mol} \%)$ and SIPr (7.56 mg, $0.019 \mathrm{mmol}, 10.0 \mathrm{~mol} \%)$ in a glove box. Subsequently, toluene $(0.19 \mathrm{~mL}, 1.0 \mathrm{M})$ and then methanol $(9.40 \mu \mathrm{L}, 0.232 \mathrm{mmol}, 1.20$ equiv) were added. The vial was sealed with a Teflon-lined screw cap, removed from the glove box, and stirred at $80{ }^{\circ} \mathrm{C}$ for $16 \mathrm{~h}$. After cooling to $23{ }^{\circ} \mathrm{C}$, the mixture was diluted with hexanes $(0.5 \mathrm{~mL})$ and filtered over a plug of silica gel (10 $\mathrm{mL}$ of EtOAc eluent). The volatiles were removed under reduced pressure and the yield was determined by ${ }^{1} \mathrm{H}$ NMR analysis with 1,3,5-trimethoxybenzene as an external standard.

\section{Any modifications of the conditions shown in the representative procedure} above are specified in Figure 2.

\section{B. Optimization and Relevant Control Experiments.}
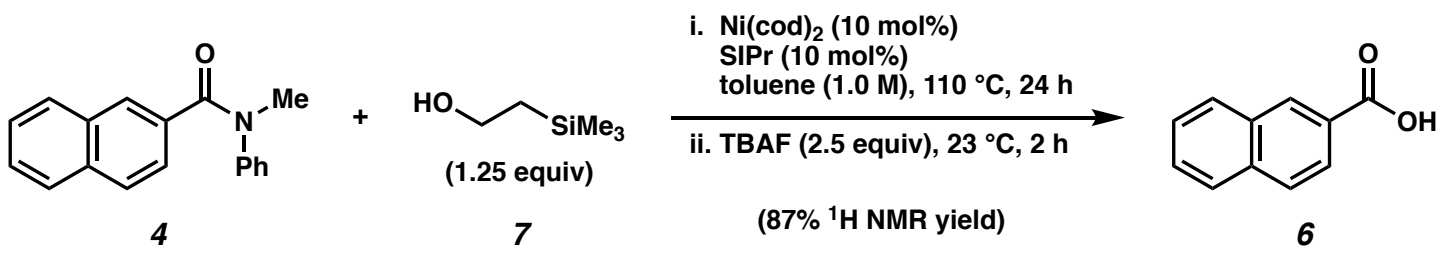

Representative Procedure for the net-hydrolysis of amides from Table 1. (Entry 3 is used as an example). A 2-dram vial containing amide 4 (40.0 $\mathrm{mg}, 0.153 \mathrm{mmol}, 1.00$ equiv) and a magnetic stir bar was charged with $\mathrm{Ni}(\operatorname{cod})_{2}(4.21 \mathrm{mg}, 0.015 \mathrm{mmol}, 10 \mathrm{~mol} \%)$ and $\mathrm{SIPr}(3.00$ $\mathrm{mg}, 0.015 \mathrm{mmol}, 10 \mathrm{~mol} \%)$ in a glove box. Subsequently, toluene $(0.15 \mathrm{~mL}, 1.0 \mathrm{M})$ and TMSethanol (7) $(27.4 \mu \mathrm{L}, 0.191 \mathrm{mmol}, 1.25$ equiv) were added. The vial was sealed with a Teflon- 
lined screw cap, removed from the glove box, and stirred at $110{ }^{\circ} \mathrm{C}$ for $24 \mathrm{~h}$. After cooling to 23 ${ }^{\circ} \mathrm{C}$, TBAF ( $0.383 \mathrm{mmol}, 2.50$ equiv) was added to the reaction mixture. The resulting mixture was stirred for $2 \mathrm{~h}$. The mixture was quenched with $1.0 \mathrm{M} \mathrm{HCl}(2.0 \mathrm{~mL})$ and diluted with water $(5 \mathrm{~mL})$. The layers were then separated and the aqueous layer was extracted with EtOAc $(3 \times 6$ $\mathrm{mL}$ ). The combined organic layers were then dried over $\mathrm{Na}_{2} \mathrm{SO}_{4}$, filtered, and concentrated under reduced pressure. The yield was determined by ${ }^{1} \mathrm{H}$ NMR analysis with 1,3,5-trimethoxybenzene as an external standard.

\section{Any modifications of the conditions shown in the representative procedure above are specified in Table 1.}

Using the representative procedure, albeit without the addition of TBAF, a series of control experiments were performed to optimize the nickel-catalyzed esterification using TMS-ethanol (7). Key results are shown in Table S1. The control experiments indicate the desired transformation is nickel-catalyzed, as no desired ester product was generated.

Table S1. Relevant Control Experiments

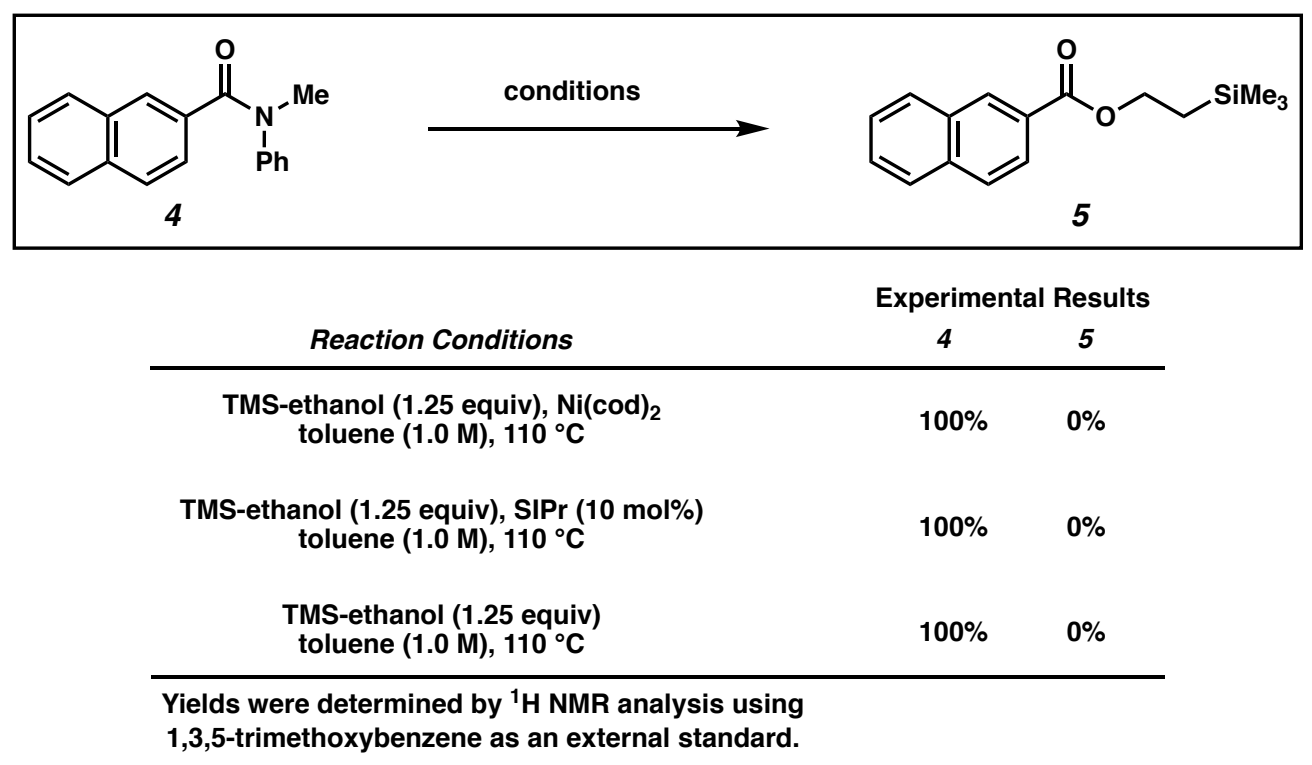




\section{Scope of Methodology}
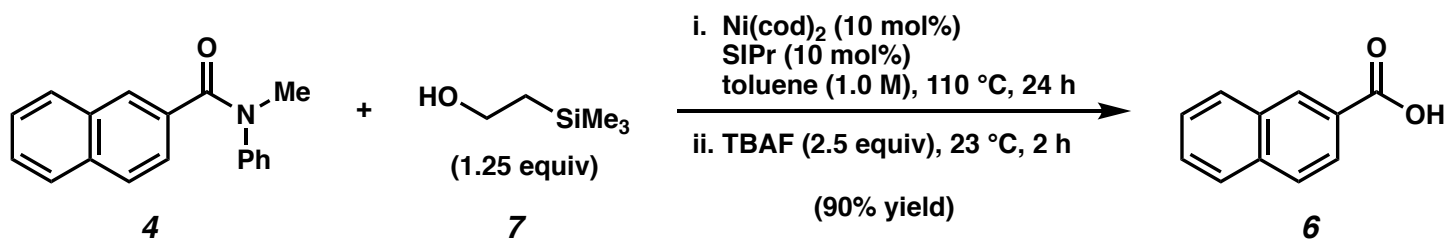

\section{Representative Procedure (net-hydrolysis of amide 4 is used as an example).}

Carboxylic Acid 6 (Figure 3). A 2-dram vial containing amide 4 (50.6 mg, 0.194 mmol, 1.0 equiv) and a magnetic stir bar was charged with $\mathrm{Ni}(\operatorname{cod})_{2}(5.33 \mathrm{mg}, 0.0194 \mathrm{mmol}, 10 \mathrm{~mol} \%)$ and $\operatorname{SIPr}(7.56 \mathrm{mg}, 0.0194 \mathrm{mmol}, 10 \mathrm{~mol} \%)$ in a glove box. Subsequently, toluene $(0.20 \mathrm{~mL}, 1.0 \mathrm{M})$ and then TMS-ethanol (34.7 $\mu \mathrm{L}, 0.242 \mathrm{mmol}, 1.25$ equiv) were added. The vial was sealed with a Teflon-lined screw cap, removed from the glove box, and stirred at $110{ }^{\circ} \mathrm{C}$ for $24 \mathrm{~h}$. After cooling to $23{ }^{\circ} \mathrm{C}$, TBAF (0.485 mmol, 2.50 equiv) was added to the reaction mixture. The resulting mixture was stirred for $2 \mathrm{~h}$. Once the reaction was complete, the mixture was quenched with $1.0 \mathrm{M} \mathrm{HCl}(3.0 \mathrm{~mL})$ and diluted with water $(5 \mathrm{~mL})$. The layers were separated and the aqueous layer was extracted with EtOAc $(3 \times 10 \mathrm{~mL})$. The combined organic layers were then dried over $\mathrm{Na}_{2} \mathrm{SO}_{4}$, filtered, and concentrated under reduced pressure. The crude residue was purified by flash chromatography (5:1 hexanes:EtOAc $+1 \%$ acetic acid) to yield carboxylic acid product 6 (90\% yield, average of two experiments) as a white solid. Carboxylic acid $6 \mathrm{R}_{f} 0.55$ (5:1 Hexanes:EtOAc $+1 \%$ acetic acid). ${ }^{1} \mathrm{H}$ NMR (600 MHz, $\left.\mathrm{CDCl}_{3}+\mathrm{DMSO}-d_{6}\right): \delta 8.59(\mathrm{~s}, 1 \mathrm{H})$, $8.08(\mathrm{dd}, J=7.2,1.4,1 \mathrm{H}), 8.02-7.97(\mathrm{~m}, 1 \mathrm{H}), 7.95-7.87(\mathrm{~m}, 2 \mathrm{H}), 7.66-7.60(\mathrm{~m}, 1 \mathrm{H}), 7.60-7.54$ $(\mathrm{m}, 1 \mathrm{H})$. Spectral data match those previously reported. ${ }^{2}$

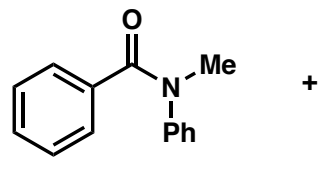

1

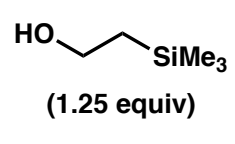

7

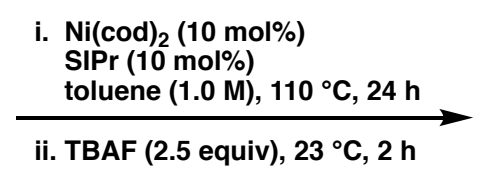

(73\% yield)

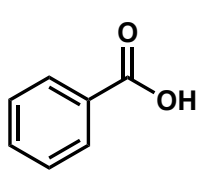

2

Carboxylic Acid 2 (Figure 3). Purification by flash chromatography (5:1 hexanes:EtOAc $+1 \%$ acetic acid) generated carboxylic acid 2 (73\% yield, average of two experiments) as a white solid. Carboxylic acid 2: $\mathrm{R}_{f} 0.22$ (2:1 Hexanes:EtOAc $+1 \%$ acetic acid). ${ }^{1} \mathrm{H}$ NMR (500 MHz, 
$\left.\mathrm{CDCl}_{3}\right): \delta 8.15(\mathrm{dd}, J=8.3,1.3,2 \mathrm{H}), 7.65-7.61(\mathrm{~m}, 1 \mathrm{H}), 7.49-7.45(\mathrm{~m}, 2 \mathrm{H})$. Spectral data match those previously reported. ${ }^{2}$
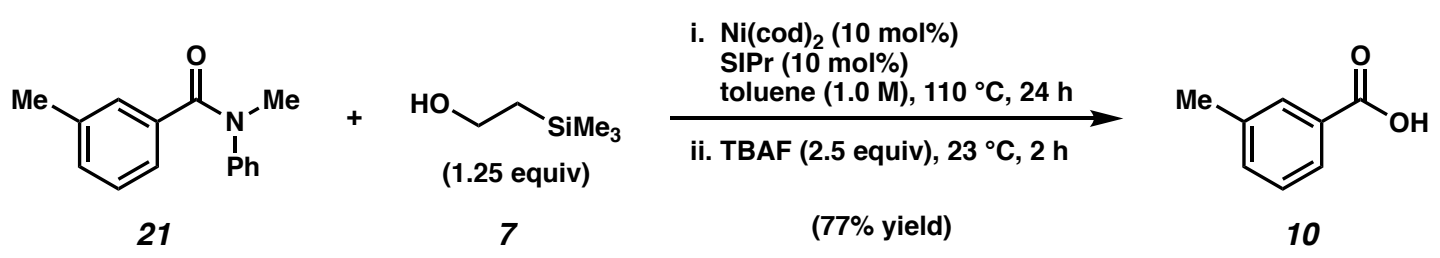

Carboxylic Acid 10 (Figure 3). Purification by flash chromatography (5:1 hexanes:EtOAc $+1 \%$ acetic acid) generated carboxylic acid 10 (77\% yield, average of two experiments) as a white solid. Carboxylic acid 10: $\mathrm{R}_{f} 0.54$ (5:1 Hexanes:EtOAc $+1 \%$ acetic acid). ${ }^{1} \mathrm{H}$ NMR (600 MHz, $\left.\mathrm{CDCl}_{3}\right): \delta$ 7.96-7.90 (m, 2H), 7.45-7.41 (m, 1H), 7.39-7.35 (m, 1H), $2.43(\mathrm{~s}, 3 \mathrm{H})$. Spectral data match those previously reported. ${ }^{3}$
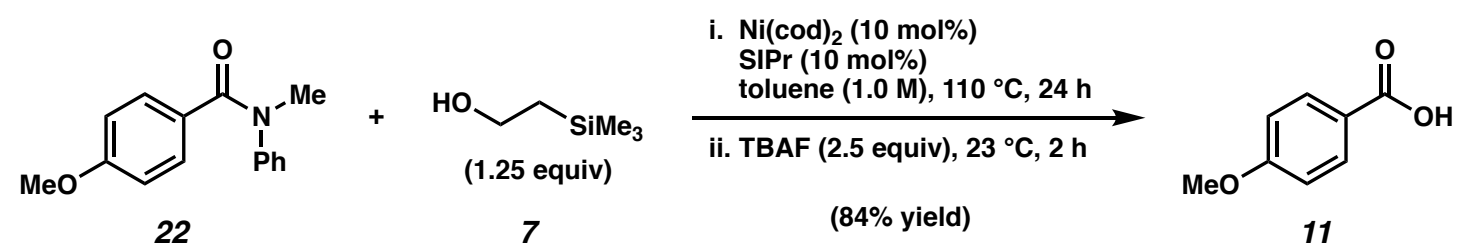

Carboxylic Acid 11 (Figure 3). Purification by flash chromatography (5:1 hexanes:EtOAc $+1 \%$ acetic acid) generated carboxylic acid 11 (84\% yield, average of two experiments) as a white solid. Carboxylic acid 11: $\mathrm{R}_{f} 0.33$ (5:1 Hexanes:EtOAc+ $1 \%$ acetic acid). ${ }^{1} \mathrm{H}$ NMR $(600 \mathrm{MHz}$, $\left.\mathrm{CDCl}_{3}\right): \delta 7.85(\mathrm{~d}, J=8.5,2 \mathrm{H}), 6.97(\mathrm{~d}, J=8.5,2 \mathrm{H}), 3.78(\mathrm{~s}, 3 \mathrm{H})$. Spectral data match those previously reported. ${ }^{2}$<smiles>CN(C(=O)c1ccc(C(F)(F)F)cc1)c1ccccc1</smiles>
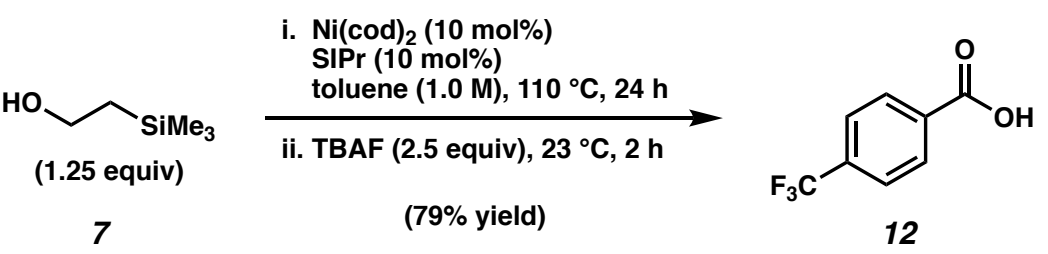

Carboxylic Acid 12 (Figure 3). Purification by flash chromatography (5:1 hexanes:EtOAc + 1\% acetic acid) generated carboxylic acid 12 (79\% yield, average of two experiments) as a white solid. Carboxylic acid 12: $\mathrm{R}_{f} 0.31$ (2:1 Hexanes:EtOAc $+1 \%$ acetic acid). ${ }^{1} \mathrm{H}$ NMR (600 MHz, 
$\left.\mathrm{CDCl}_{3}\right): \delta 8.10(\mathrm{~d}, J=8.1,2 \mathrm{H}), 7.84(\mathrm{~d}, J=8.6,2 \mathrm{H})$. Spectral data match those previously reported. $^{2}$

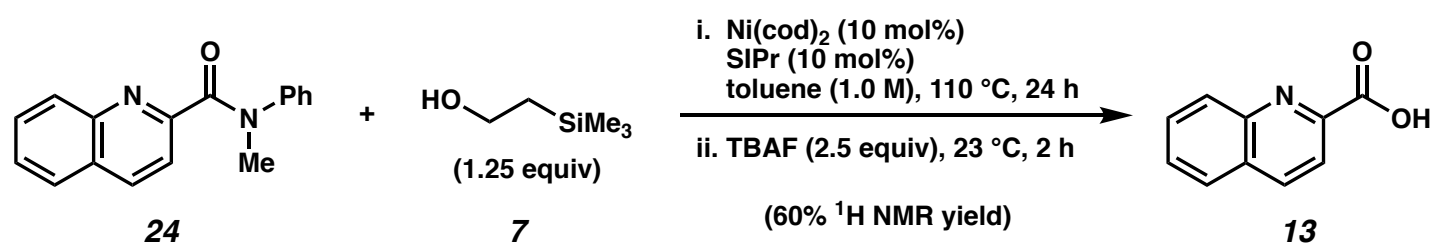

Carboxylic Acid 13 (Figure 3). ${ }^{1} \mathrm{H}$ NMR $\left(600 \mathrm{MHz}, \mathrm{CDCl}_{3}\right)$ of crude reaction mixture: $\delta 8.13$ $(\mathrm{d}, J=8.25,1 \mathrm{H}), 7.93(\mathrm{~d}, J=8.25,1 \mathrm{H}), 7.85(\mathrm{~d}, J=7.66,1 \mathrm{H}), 7.81(\mathrm{~d}, J=8.25,1 \mathrm{H}), 7.67-7.63$ $(\mathrm{m}, 1 \mathrm{H}), 7.75-7.46(\mathrm{~m}, 1 \mathrm{H})$. Spectral data of the crude mixture of carboxylic acid $\mathbf{1 3}$ match those previously reported. ${ }^{4}$

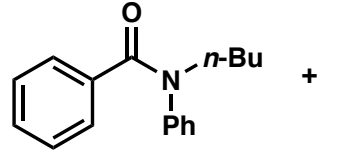

$14 a$

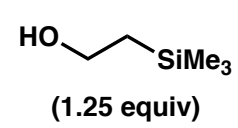

7

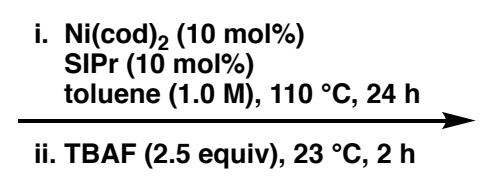

( $77 \%$ yield)

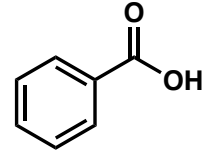

2

Carboxylic Acid 2 (Table 2). Purification by preparative thin-layer chromatography (5:1 Benzene:EtOAc $+0.1 \%$ acetic acid) generated carboxylic acid 2 (77\% yield, average of two experiments) as a white solid. Carboxylic acid 2: $\mathrm{R}_{f} 0.22$ (2:1 Hexanes:EtOAc $+1 \%$ acetic acid). ${ }^{1} \mathrm{H}$ NMR $\left(500 \mathrm{MHz}, \mathrm{CDCl}_{3}\right): \delta 8.15(\mathrm{dd}, J=8.3,1.3,2 \mathrm{H}), 7.65-7.61(\mathrm{~m}, 1 \mathrm{H}), 7.49-7.45(\mathrm{~m}$, $2 \mathrm{H})$. Spectral data match those previously reported. ${ }^{2}$

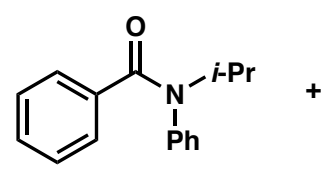

$14 b$

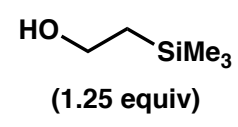

7

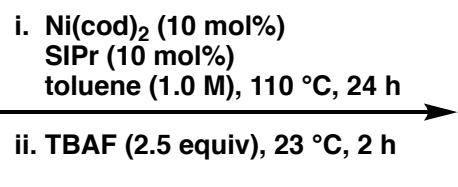

$(60 \%$ yield $)$

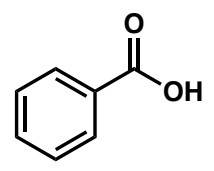

2

Carboxylic Acid 2 (Table 2). Purification by preparative thin-layer chromatography (5:1 Benzene:EtOAc $+0.1 \%$ acetic acid) generated carboxylic acid 2 (60\% yield, average of two experiments) as a white solid. Carboxylic acid 2: $\mathrm{R}_{f} 0.22$ (2:1 Hexanes:EtOAc $+1 \%$ acetic acid). ${ }^{1} \mathrm{H}$ NMR $\left(500 \mathrm{MHz}, \mathrm{CDCl}_{3}\right): \delta 8.15(\mathrm{dd}, J=8.3,1.3,2 \mathrm{H}), 7.65-7.61(\mathrm{~m}, 1 \mathrm{H}), 7.49-7.45(\mathrm{~m}$, $2 \mathrm{H})$. Spectral data match those previously reported. ${ }^{2}$ 


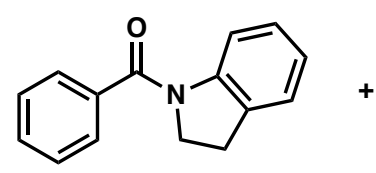

$14 c$

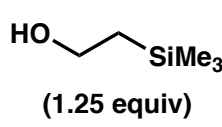

7 i. $\mathrm{Ni}(\operatorname{cod})_{2}(10 \mathrm{~mol} \%)$

SIPr (10 mol\%)

toluene $(1.0 \mathrm{M}), 110^{\circ} \mathrm{C}, 24 \mathrm{~h}$

ii. TBAF ( 2.5 equiv), $23^{\circ} \mathrm{C}, 2 \mathrm{~h}$

$(56 \%$ yield)<smiles>O=C(O)c1ccccc1</smiles>

2

Carboxylic Acid 2 (Table 2). Purification by preparative thin-layer chromatography (1:1:3 Benzene:EtOAc:DCM) generated carboxylic acid 2 (56\% yield, average of two experiments) as a white solid. Carboxylic acid 2: $\mathrm{R}_{f} 0.22$ (2:1 Hexanes:EtOAc $+1 \%$ acetic acid). ${ }^{1} \mathrm{H}$ NMR (500 $\left.\mathrm{MHz}, \mathrm{CDCl}_{3}\right): \delta 8.15(\mathrm{dd}, J=8.3,1.3,2 \mathrm{H}), 7.65-7.61(\mathrm{~m}, 1 \mathrm{H}), 7.49-7.45(\mathrm{~m}, 2 \mathrm{H})$. Spectral data match those previously reported. ${ }^{2}$<smiles>NN([As])C(=O)c1ccccc1</smiles>

14d

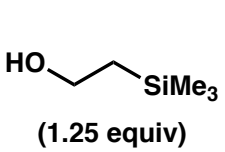

7

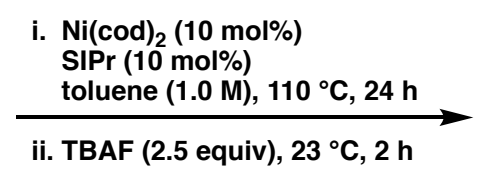

(71\% yield)

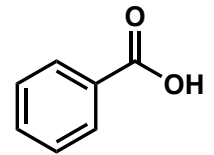

2

Carboxylic Acid 2 (Table 2). Purification by preparative thin-layer chromatography (1:1:2 Benzene:EtOAc:DCM) generated carboxylic acid 2 (71\% yield, average of two experiments) as a white solid. Carboxylic acid 2: $\mathrm{R}_{f} 0.22$ (2:1 Hexanes:EtOAc $+1 \%$ acetic acid). ${ }^{1} \mathrm{H}$ NMR (500 $\left.\mathrm{MHz}, \mathrm{CDCl}_{3}\right): \delta 8.15(\mathrm{dd}, J=8.3,1.3,2 \mathrm{H}), 7.65-7.61(\mathrm{~m}, 1 \mathrm{H}), 7.49-7.45(\mathrm{~m}, 2 \mathrm{H})$. Spectral data match those previously reported. ${ }^{2}$

\section{1.0 mmol-Scale Reaction}

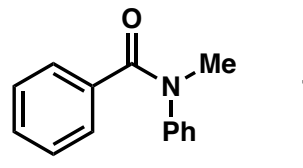

1

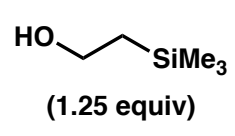

7

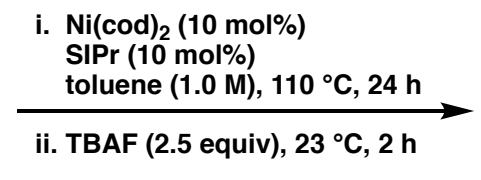

(72\% yield)<smiles>O=C(O)c1ccccc1</smiles>

2

Carboxylic Acid 2 (Table 2). A 2-dram vial containing amide 1 (220 mg, 1.0 mmol, 1.0 equiv) and a magnetic stir bar was charged with $\mathrm{Ni}(\operatorname{cod})_{2}(28.6 \mathrm{mg}, 0.10 \mathrm{mmol}, 10 \mathrm{~mol} \%)$ and $\mathrm{SIPr}$ (40.7 mg, $0.10 \mathrm{mmol}, 10 \mathrm{~mol} \%)$ in a glove box. Subsequently, toluene $(1.0 \mathrm{~mL}, 1.0 \mathrm{M})$ and then TMS-ethanol (187 $\mu \mathrm{L}, 1.25 \mathrm{mmol}, 1.25$ equiv) were added. The vial was sealed with a Teflonlined screw cap, removed from the glove box, and stirred at $110{ }^{\circ} \mathrm{C}$ for $24 \mathrm{~h}$. After cooling to 23 
${ }^{\circ} \mathrm{C}$, TBAF (2.50 mmol, 2.50 equiv) was added to the reaction mixture. The resulting mixture was stirred for $2 \mathrm{~h}$. Once the reaction was complete, the mixture was quenched with $1.0 \mathrm{M} \mathrm{HCl}(5.0$ $\mathrm{mL})$ and diluted with water $(10 \mathrm{~mL})$. The layers were separated and the aqueous layer was extracted with EtOAc $(3 \times 15 \mathrm{~mL})$. The combined organic layers were then dried over $\mathrm{Na}_{2} \mathrm{SO}_{4}$, filtered, and concentrated under reduced pressure. The crude residue was purified by flash chromatography (20:1 hexanes:EtOAc $+1 \%$ acetic acid) to yield carboxylic acid product $\mathbf{2}$ ( $72 \%$ yield, average of two experiments) as a white solid. Spectral data match those previously reported for carboxylic acid 2 (see page S5).

\section{E. Competition Experiments}

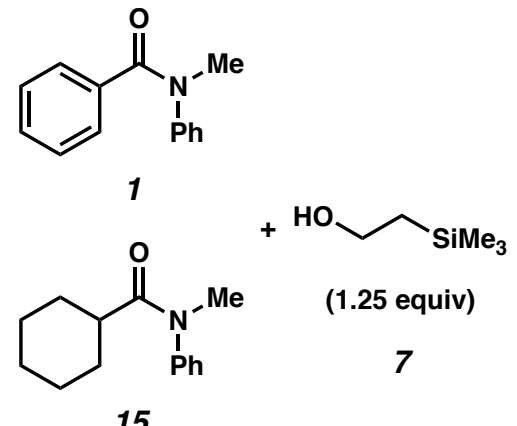

15 i. $\mathrm{Ni}(\operatorname{cod})_{2}(10 \mathrm{~mol} \%)$ $\operatorname{SIPr}(10 \mathrm{~mol} \%)$ toluene $(1.0 \mathrm{M}), 110^{\circ} \mathrm{C}, 24 \mathrm{~h}$ ii. TBAF ( 2.5 equiv), $23^{\circ} \mathrm{C}, 2 \mathrm{~h}$

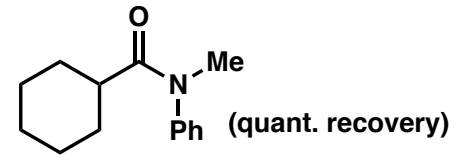

15

Selectivity for Aromatic over Aliphatic Amides (Figure 4). A 2-dram vial containing amide 1 (52.4 mg, $0.248 \mathrm{mmol}, 1.0$ equiv), amide 15 (53.9 mg, $0.248 \mathrm{mmol}, 1.0$ equiv) and a magnetic stir bar was charged with $\mathrm{Ni}(\operatorname{cod})_{2}(6.82 \mathrm{mg}, 0.025 \mathrm{mmol}, 10 \mathrm{~mol} \%)$ and $\mathrm{SIPr}(9.69 \mathrm{mg}, 0.025$ $\mathrm{mmol}, 10 \mathrm{~mol} \%)$ in a glove box. Subsequently, toluene $(0.25 \mathrm{~mL}, 1.0 \mathrm{M})$ and then TMS-ethanol (44.4 $\mu \mathrm{L}, 0.310 \mathrm{mmol}, 1.25$ equiv) were added. The vial was sealed with a Teflon-lined screw cap, removed from the glove box, and stirred at $110^{\circ} \mathrm{C}$ for $24 \mathrm{~h}$. After cooling to $23{ }^{\circ} \mathrm{C}$, TBAF ( $0.620 \mathrm{mmol}, 2.5$ equiv) was added to the reaction mixture. The resulting mixture was stirred for $2 \mathrm{~h}$. The mixture was quenched with $1.0 \mathrm{M} \mathrm{HCl}(3.0 \mathrm{~mL})$ and diluted with water $(5 \mathrm{~mL})$. The layers were separated and the aqueous layer was extracted with EtOAc $(3 \times 10 \mathrm{~mL})$. The combined organic layers were then dried over $\mathrm{Na}_{2} \mathrm{SO}_{4}$, filtered, and concentrated under reduced pressure. Spectral data match those previously reported for carboxylic acid 2 (see page S5). 

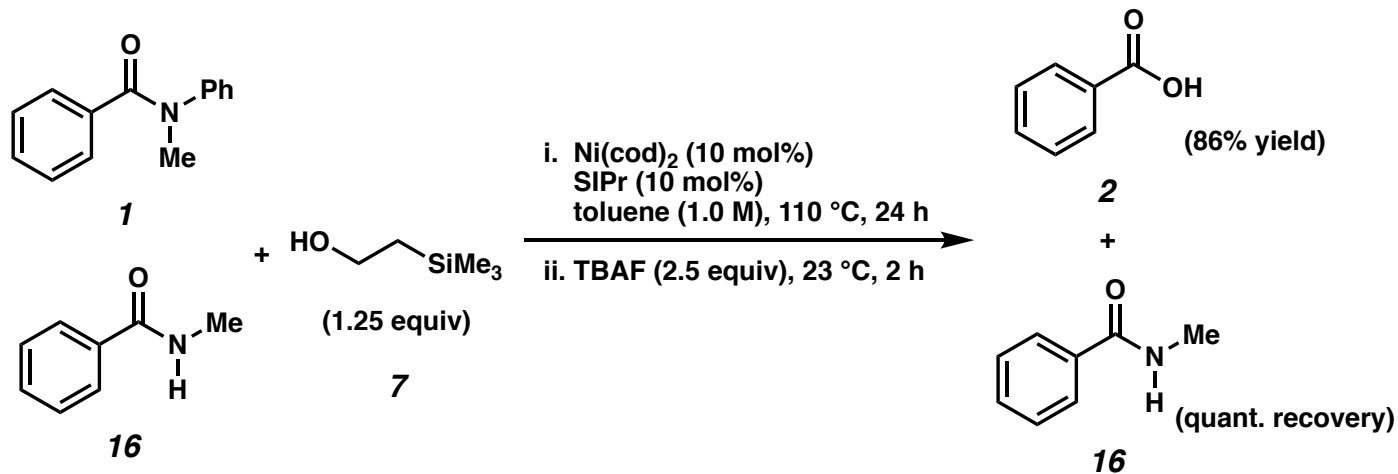

Selectivity for Tertiary over Secondary Amides (Figure 4). A 2-dram vial containing amide 1 (54.0 mg, $0.256 \mathrm{mmol}, 1.0$ equiv), amide 16 (34.2 $\mathrm{mg}, 0.253 \mathrm{mmol}, 0.99$ equiv) and a magnetic stir bar was charged with $\mathrm{Ni}(\mathrm{cod})_{2}(7.03 \mathrm{mg}, 0.026 \mathrm{mmol}, 10 \mathrm{~mol} \%)$ and $\mathrm{SIPr}(9.98 \mathrm{mg}, 0.026$ $\mathrm{mmol}, 10 \mathrm{~mol} \%)$ in a glove box. Subsequently, toluene $(0.26 \mathrm{~mL}, 1.0 \mathrm{M})$ and then TMS-ethanol $(45.8 \mu \mathrm{L}, 0.320 \mathrm{mmol}, 1.25$ equiv) were added. The vial was sealed with a Teflon-lined screw cap, removed from the glove box, and stirred at $110{ }^{\circ} \mathrm{C}$ for $24 \mathrm{~h}$. After cooling to $23{ }^{\circ} \mathrm{C}$, TBAF ( $0.64 \mathrm{~mL}, 0.639 \mathrm{mmol}, 2.50$ equiv) was added to the reaction mixture via syringe. The resulting mixture was stirred at $23{ }^{\circ} \mathrm{C}$ for $2 \mathrm{~h}$. Then, the mixture was quenched with $1.0 \mathrm{M} \mathrm{HCl}(4.0 \mathrm{~mL})$ and diluted with water $(3 \mathrm{~mL})$. The layers were separated and the aqueous layer was extracted with EtOAc ( $3 \times 10 \mathrm{~mL})$. The combined organic layers were then washed with saturated $\mathrm{NaCl}$ (15 mL), dried over $\mathrm{Na}_{2} \mathrm{SO}_{4}$, filtered, and concentrated under reduced pressure. Spectral data match those previously reported for carboxylic acid $\mathbf{2}$ (see page S5).

\section{F. Selective Cleavage of Aryl Amide in the Presence of an Ester}
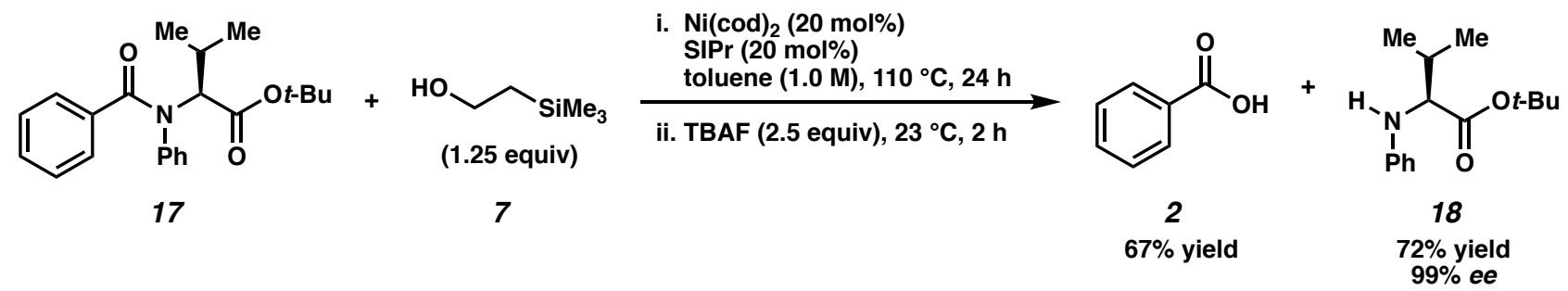

Carboxylic Acid 2 and Amine 18 (Figure 5). A 2-dram vial containing amide 17 (50.9 mg, $0.144 \mathrm{mmol}, 1.0$ equiv) and a magnetic stir bar was charged with $\mathrm{Ni}(\mathrm{cod})_{2}(7.92 \mathrm{mg}, 0.029$ mmol, $20 \mathrm{~mol} \%)$ and SIPr (11.3 $\mathrm{mg}, 0.029 \mathrm{mmol}, 20 \mathrm{~mol} \%)$ in a glove box. Subsequently, toluene $(0.14 \mathrm{~mL}, 1.0 \mathrm{M})$ and then TMS-ethanol $(25.8 \mu \mathrm{L}, 0.180 \mathrm{mmol}, 1.25$ equiv) were added. 
The vial was sealed with a Teflon-lined screw cap, removed from the glove box, and stirred at $110{ }^{\circ} \mathrm{C}$ for $24 \mathrm{~h}$. After cooling to $23{ }^{\circ} \mathrm{C}$, TBAF ( $0.360 \mathrm{mmol}$, 2.5 equiv) was added to the reaction mixture. The resulting mixture was stirred for $2 \mathrm{~h}$. Then, the mixture was quenched with water $(6.0 \mathrm{~mL})$ and diluted with water $(5 \mathrm{~mL})$. The layers were separated and the aqueous layer was extracted with EtOAc $(3 \times 10 \mathrm{~mL})$. The combined organic layers were then dried over $\mathrm{Na}_{2} \mathrm{SO}_{4}$, filtered, and concentrated under reduced pressure. The crude residue was purified by preparative thin-layer chromatography (5:1 hexanes:EtOAc $+1 \%$ acetic acid) to yield carboxylic acid product 2 (67\% yield, average of two experiments) as a white solid and amine 18 (72\% yield, $99 \% e e$, average of two experiments) as a clear oil. Spectral data matched those previously reported. ${ }^{1}$ Carboxylic acid 2: $\mathrm{R}_{f} 0.22$ (2:1 Hexanes:EtOAc $+1 \%$ acetic acid). Amine 18: $\mathrm{R}_{f} 0.24$ (9:1 Hexanes:EtOAc). ${ }^{1} \mathrm{H}$ NMR (500 MHz, $\left.\mathrm{CDCl}_{3}\right): \delta 7.16(\mathrm{~m}, 2 \mathrm{H}), 6.71(\mathrm{~m}, 1 \mathrm{H}), 6.63(\mathrm{~m}, 2 \mathrm{H})$, 4.14 (br s, 1H), 3.75 (m, 1H), 2.09 (o, $J=5.6,1.1,1 \mathrm{H}), 1.43$ (s, 9H), 1.03 (dd, $J=6.7,4.3,6 \mathrm{H})$. Spectral data match those previously reported. ${ }^{1}$ 


\section{References}

${ }^{1}$ Hie, L.; Fine, N.; Shah, T. K.; Baker, E. L.; Hong, X.; Yang, Y.; Liu, P.; Houk, K. N.; Garg, N. $\mathrm{K}$. Conversion of amides to esters by the nickel-catalyzed activation of amide $\mathrm{C}-\mathrm{N}$ bonds. Nature 2015, 524, 79-83.

${ }^{2}$ Bhunia, S. K.; Das, P.; Nandi, S.; Jana, R. Carboxylation of aryl trilates with $\mathrm{CO}_{2}$ merging palladium and visible-light-photoredox catalysts. Org. Lett. 2019, 21, 4632-463.

${ }^{3}$ Kobaysashi, K.; Kondo, Y. Transition-metal-free carboxylation of organozinc reagents using $\mathrm{CO}_{2}$ in DMF solvent. Org. Lett. 2009, 11, 2035-2037.

${ }^{4}$ Yoshida, M.; Katagiri, Y.; Zhu, W.; Shishido, K. Oxidative carboxylation of arylaldehydes with water by a sulfoxylalkyl-substituted $N$-heterocyclic carbenecatalyst. Org. Biomol. Chem. 2009, 7, 4062-4066. 
Knapp et al.: Nickel-Catalyzed Conversion of Amides to Carboxylic Acids-S13

\section{${ }^{1}$ H NMR Spectra}



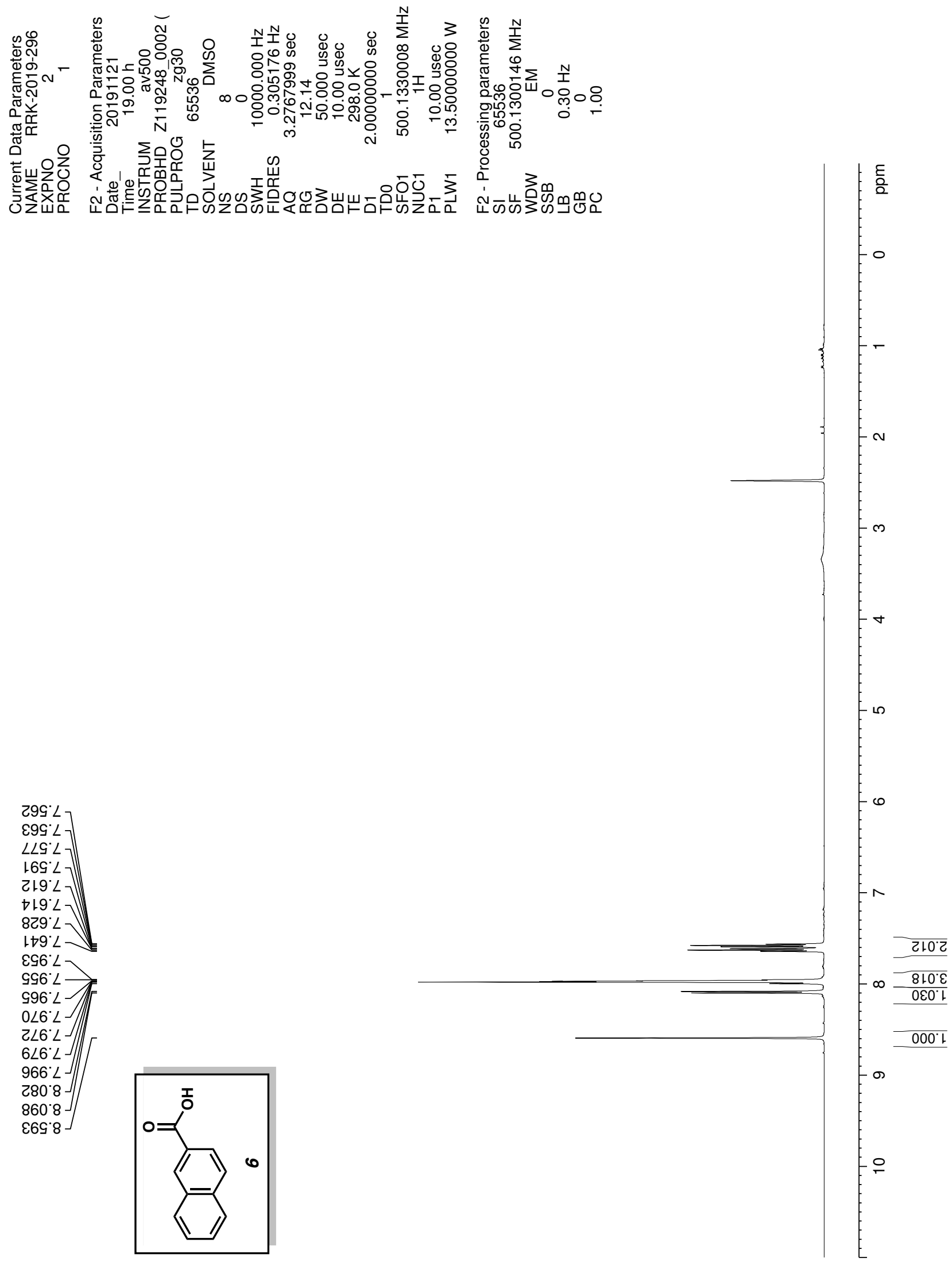

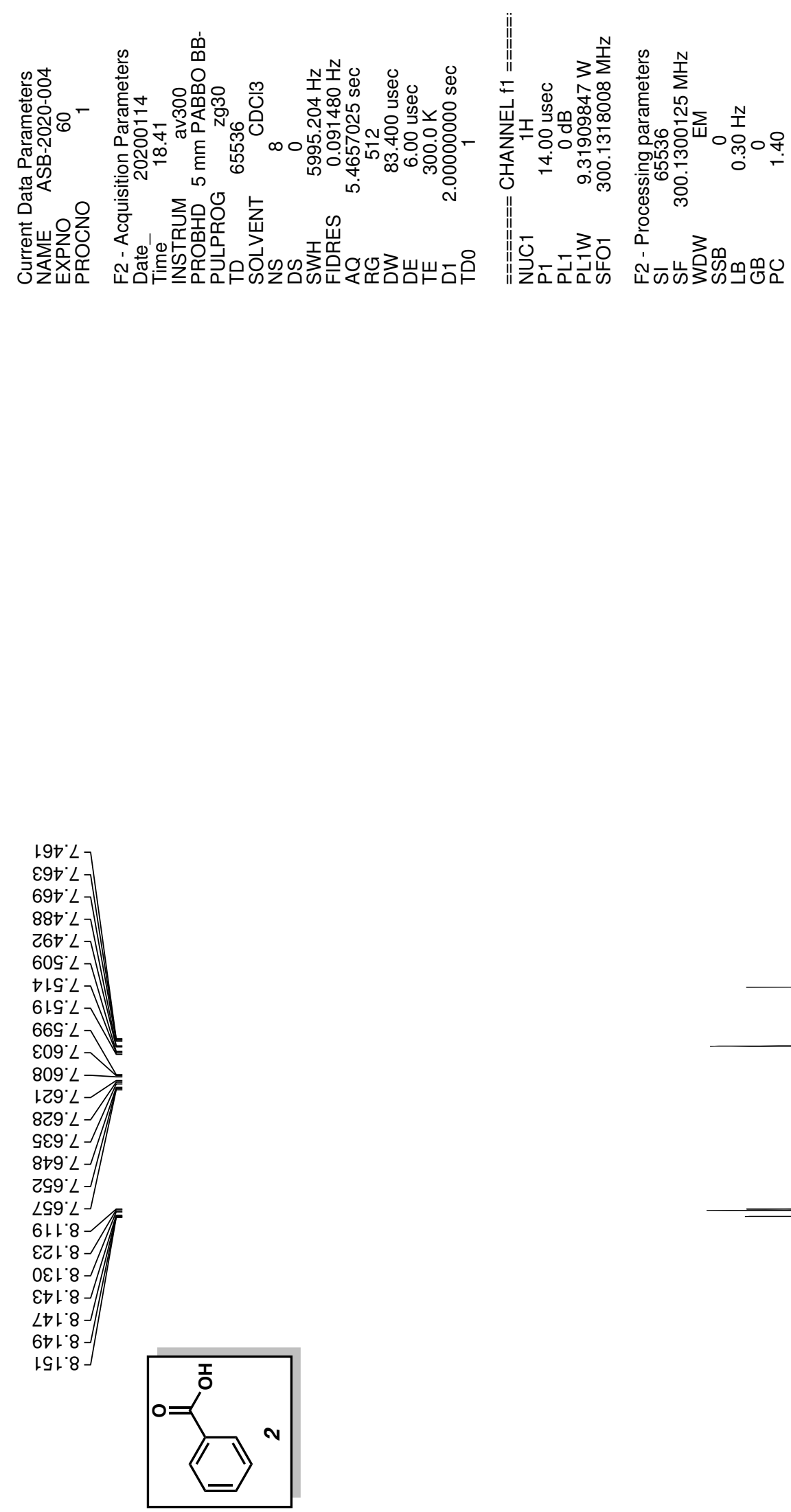


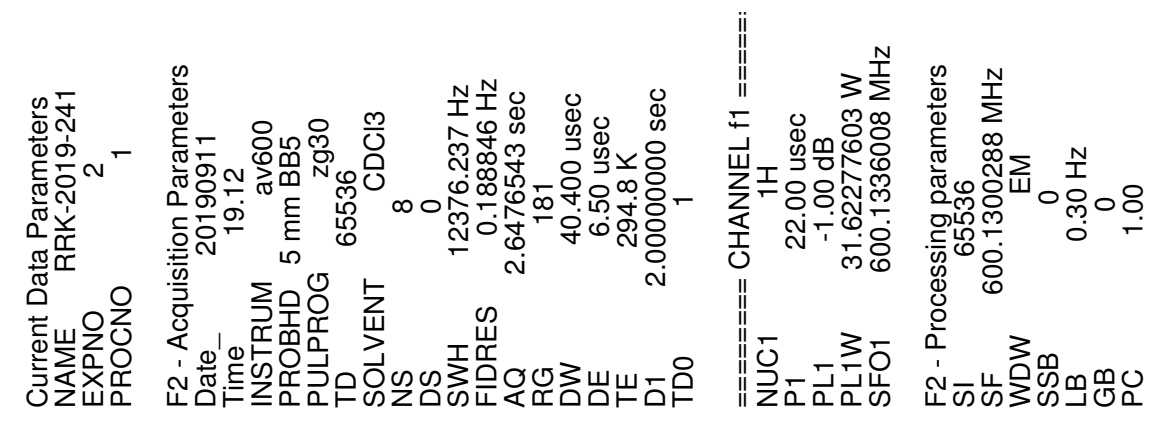

$1+6 . \angle J$

$2 \nabla 6 . \mathrm{L}$

$\varepsilon \nabla 6 \circ$

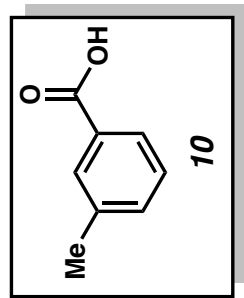




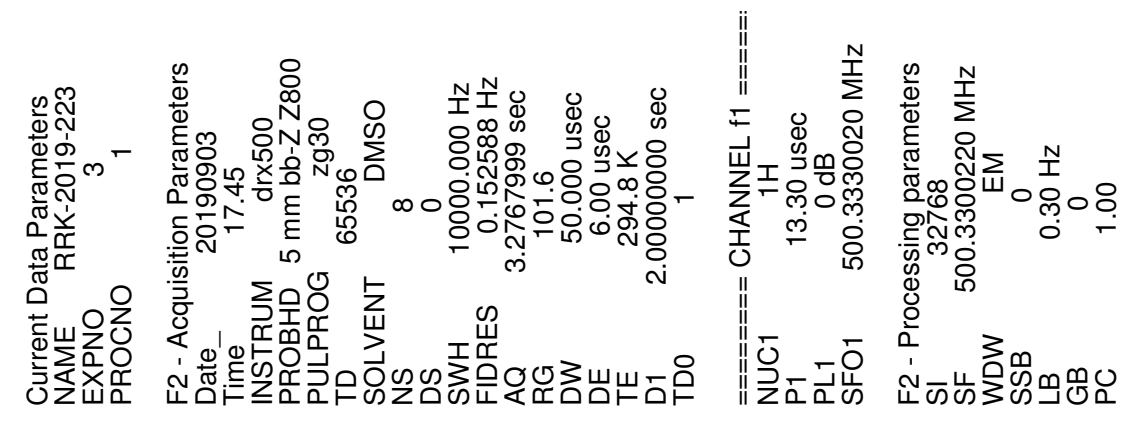

$996^{\circ} 9$

$\neg 86^{\circ} 9>$

$\nabla 78^{\circ} \mathrm{L}$

$298^{\circ} L$

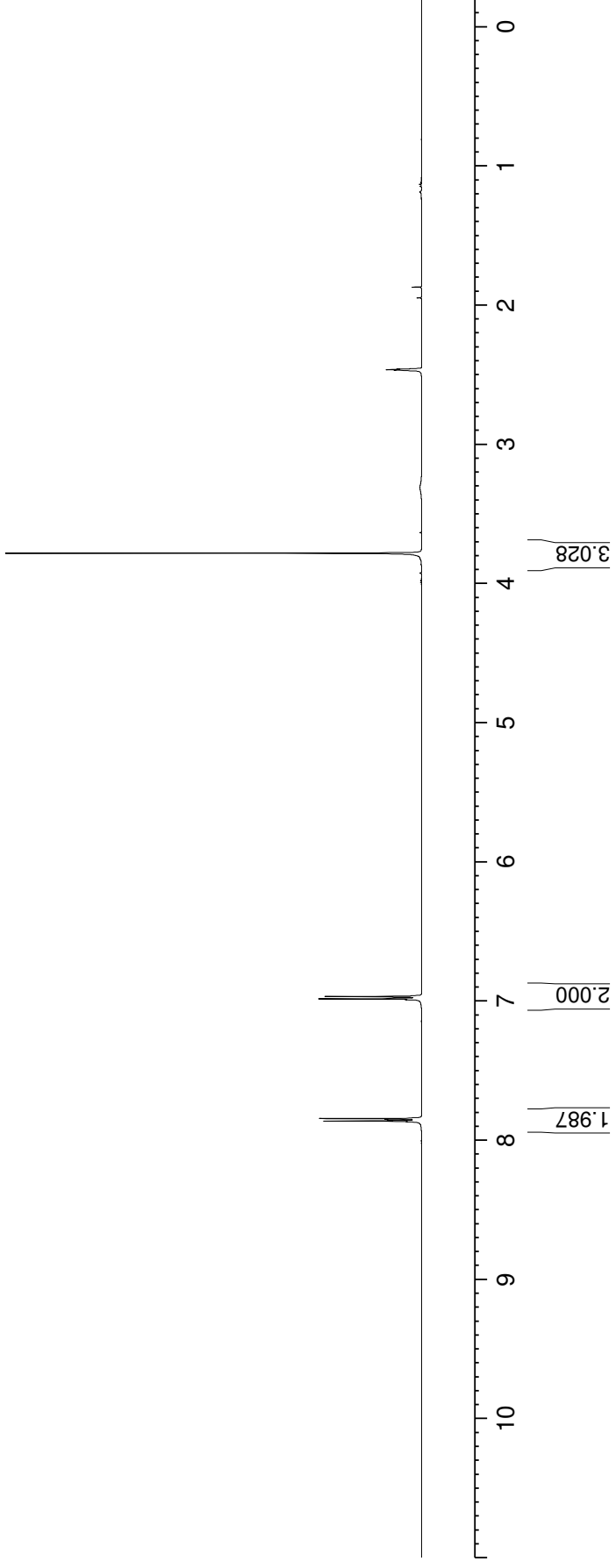




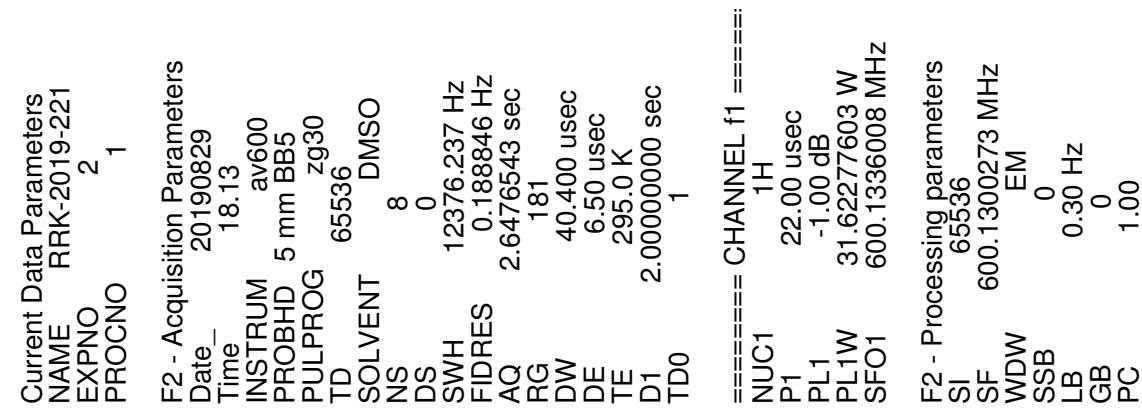

$1 E 8 \cdot L$ $778^{\circ} L>$

860.8

เレ・8
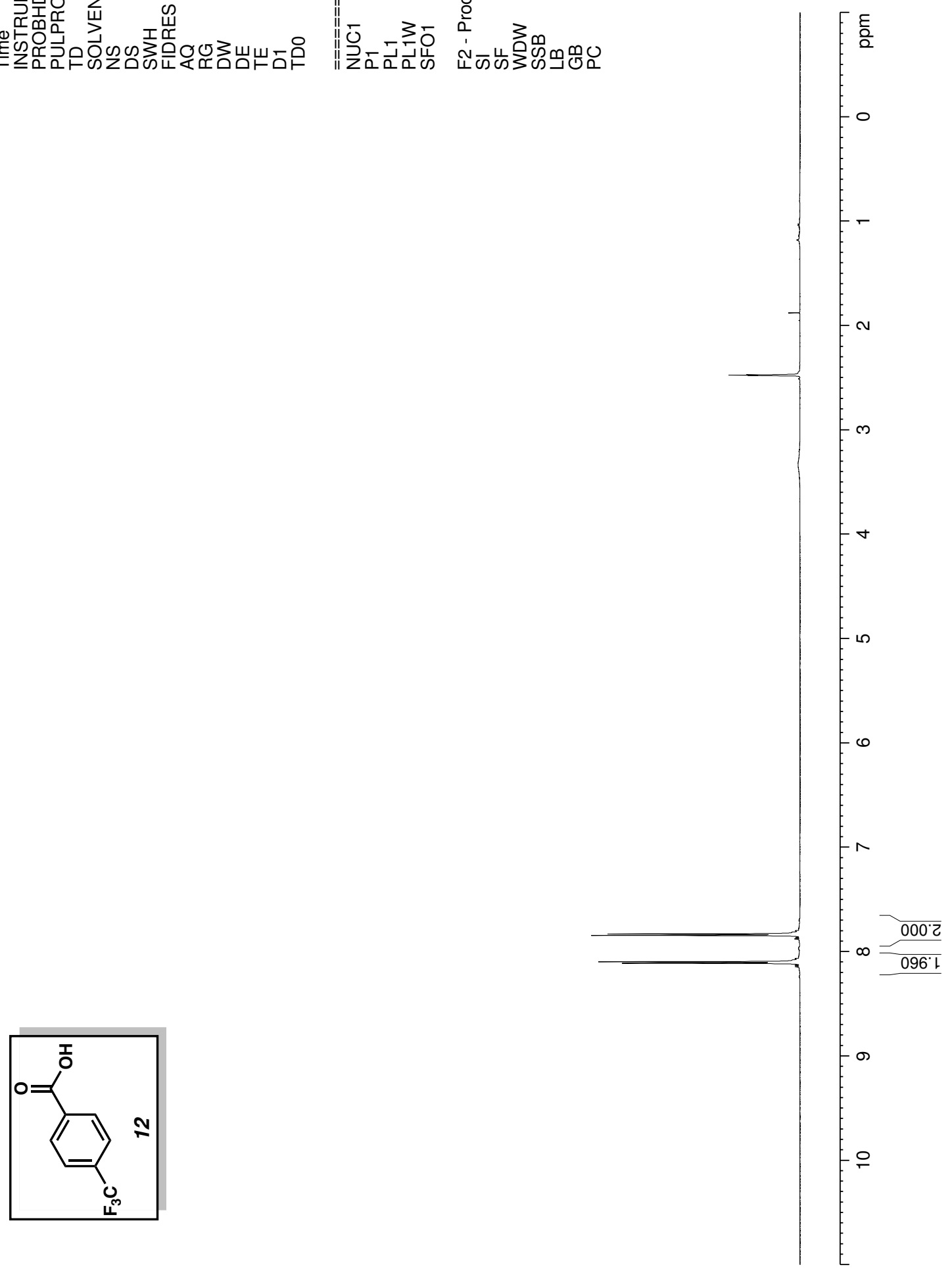
Knapp et al.: Nickel-Catalyzed Conversion of Amides to Carboxylic Acids-S19
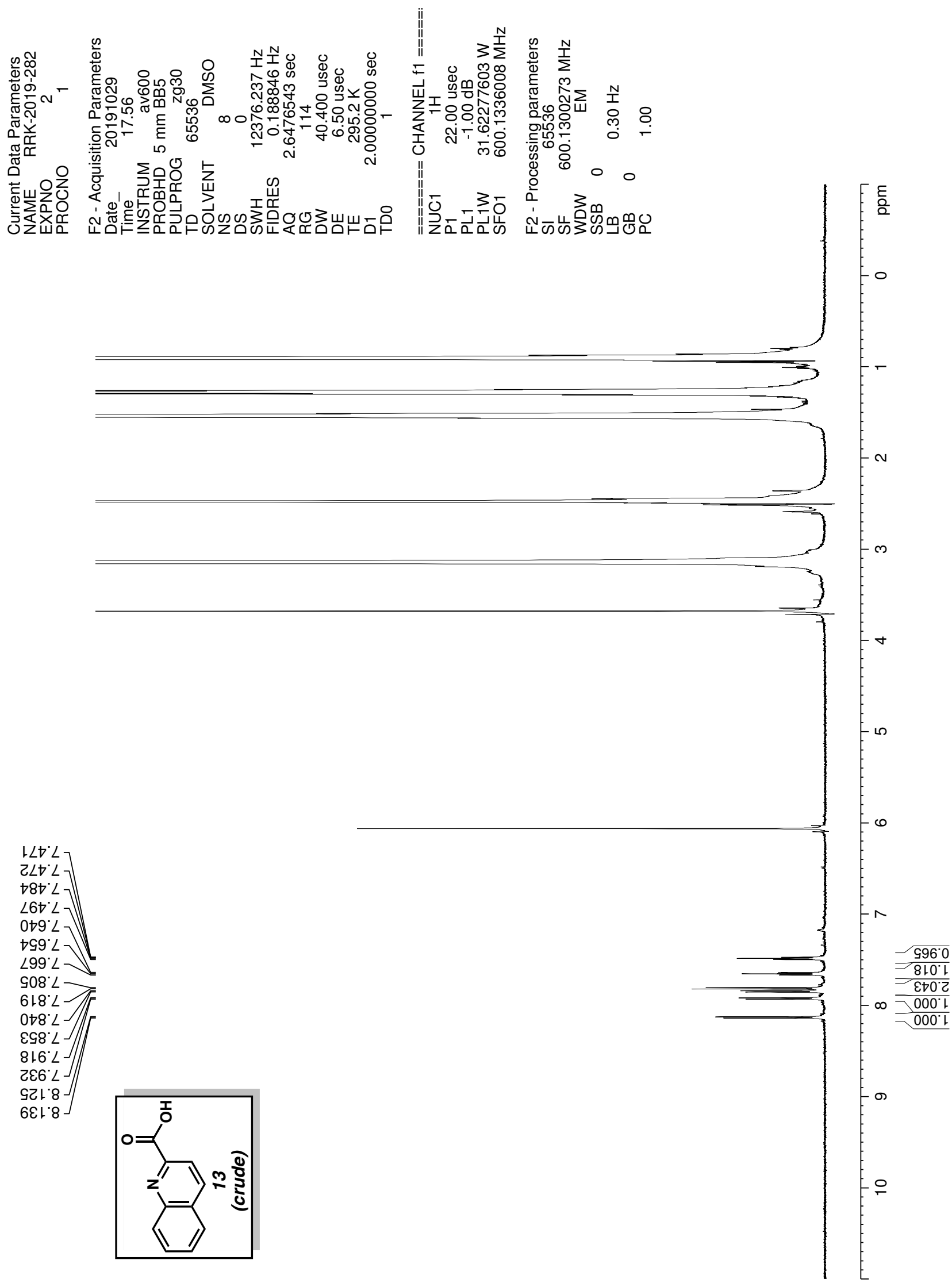

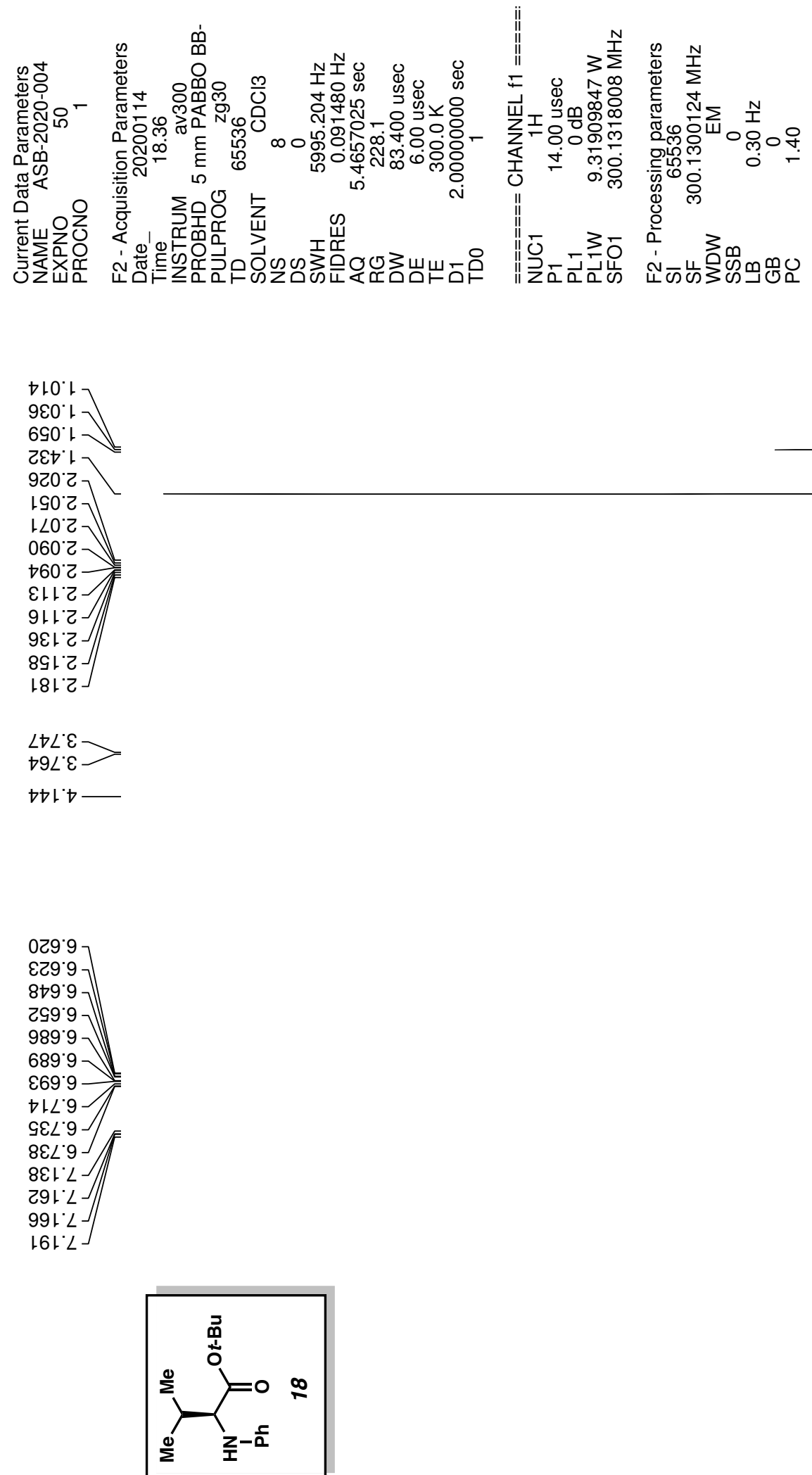


\section{SFC Traces}




\begin{tabular}{|c|c|c|c|c|c|}
\hline Compound & $\begin{array}{c}\text { SFC Method } \\
\text { Column/Temp. } \\
\text { Abs. Wavelength }\end{array}$ & Sovlent & $\begin{array}{l}\text { Method } \\
\text { Flow Rate }\end{array}$ & $\begin{array}{l}\text { Retention } \\
\text { Times } \\
\text { (min) }\end{array}$ & $\begin{array}{c}\text { Enantiomeric } \\
\text { Ratio } \\
\text { (er) }\end{array}$ \\
\hline$\left.\overbrace{\text { rac-18 }}^{\mathrm{Me}}\right|_{\mathrm{Ph}} ^{\mathrm{Me}}$ & $\begin{array}{c}\text { Daicel ChiralPak } \\
\text { IA-3/35 }{ }^{\circ} \mathrm{C} \\
\lambda \text { abs }=210 \mathrm{~nm}\end{array}$ & $\begin{array}{c}2 \% \\
\text { isopropanol in } \\
\mathrm{CO}_{2}\end{array}$ & $3.5 \mathrm{~mL} / \mathrm{min}$ & $2.20 / 2.75$ & $50: 50$ \\
\hline$\underbrace{M e}_{18}$ & $\begin{array}{c}\text { Daicel ChiralPak } \\
\text { IA-3/35 }{ }^{\circ} \mathrm{C} \\
\lambda \text { abs }=210 \mathrm{~nm}\end{array}$ & $\begin{array}{c}2 \% \\
\text { isopropanol in } \\
\mathrm{CO}_{2}\end{array}$ & $3.5 \mathrm{~mL} / \mathrm{min}$ & $2.35 / 2.87$ & $100: 0$ \\
\hline
\end{tabular}

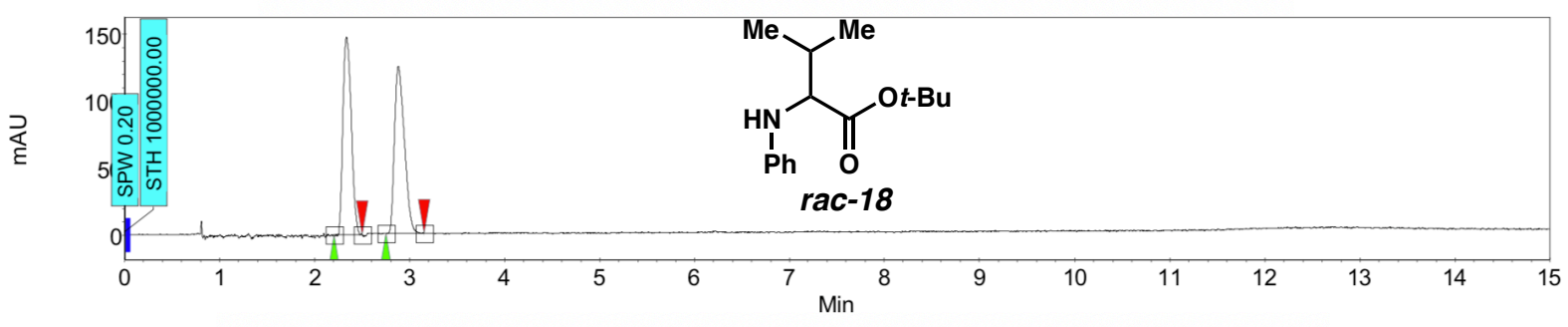

\begin{tabular}{|c|l|r|r|r|r|r|r|r|r|}
\hline Index & Name & Start & Time & End & RT Offset & Quantity & Height & Area & Area \\
\hline \hline & & {$[\mathrm{Min}]$} & {$[\mathrm{Min}]$} & {$[\mathrm{Min}]$} & {$[\mathrm{Min}]$} & {$[\%$ Area $]$} & {$[\mu \mathrm{V}]$} & {$[\mu \mathrm{V} . \mathrm{Min}]$} & {$[\%]$} \\
\hline 2 & UNKNOWN & 2.20 & 2.33 & 2.50 & 0.00 & 50.06 & 147.5 & 15.1 & 50.060 \\
\hline 1 & UNKNOWN & 2.75 & 2.88 & 3.15 & 0.00 & 49.94 & 124.8 & 15.0 & 49.940 \\
\hline & & & & & & & & & \\
\hline Total & & & & & & 100.00 & 272.3 & 30.1 & 100.000 \\
\hline
\end{tabular}




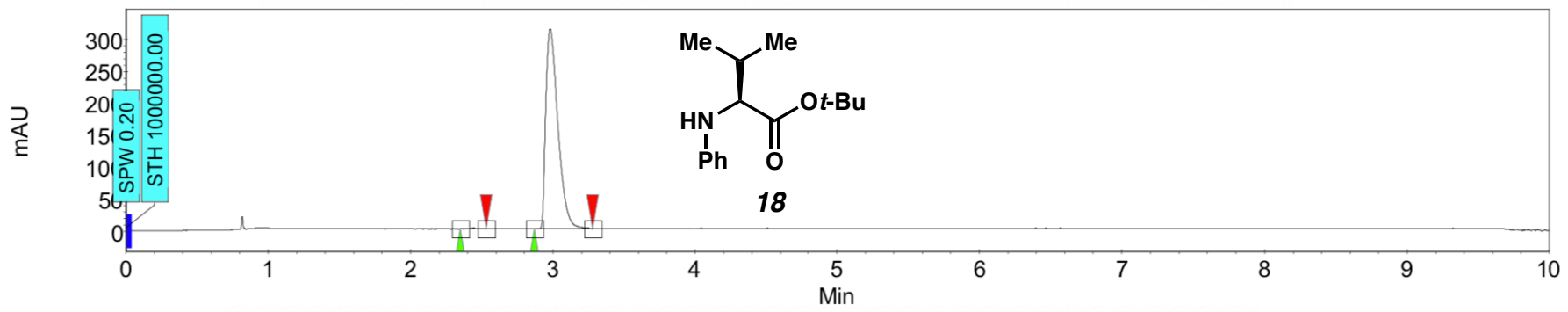

\begin{tabular}{|c|l|r|r|r|r|r|r|r|r|}
\hline Index & Name & Start & Time & End & RT Offset & Quantity & Height & Area & Area \\
\hline \hline & & {$[$ Min] } & {$[$ Min] } & {$[$ Min] } & {$[$ Min] } & {$[\%$ Area] } & {$[\mu \mathrm{V}]$} & {$[\mu \mathrm{V} . \mathrm{Min}]$} & {$[\%]$} \\
\hline 2 & UNKNOWN & 2.35 & 2.44 & 2.53 & 0.00 & 0.16 & 0.9 & 0.0 & 0.161 \\
\hline 1 & UNKNOWN & 2.87 & 2.98 & 3.28 & 0.00 & 99.84 & 312.3 & 30.7 & 99.839 \\
\hline & & & & & & & & & \\
\hline Total & & & & & & 100.00 & 313.2 & 30.8 & 100.000 \\
\hline
\end{tabular}

